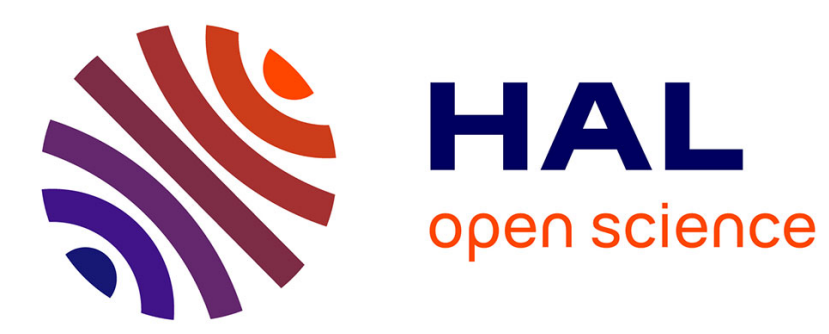

\title{
Large Amplitude Vibrations of Thin Hyperelastic Plates: Neo-Hookean Model
}

Ivan Breslavsky, Marco Amabili, Mathias Legrand

\section{To cite this version:}

Ivan Breslavsky, Marco Amabili, Mathias Legrand. Large Amplitude Vibrations of Thin Hyperelastic Plates: Neo-Hookean Model. ASME 2013 International Mechanical Engineering Congress and Exposition, Nov 2013, San Diego, United States. 10.1115/IMECE2013-62253 . hal-01933743

\section{HAL Id: hal-01933743 https://hal.science/hal-01933743}

Submitted on 24 Nov 2018

HAL is a multi-disciplinary open access archive for the deposit and dissemination of scientific research documents, whether they are published or not. The documents may come from teaching and research institutions in France or abroad, or from public or private research centers.
L'archive ouverte pluridisciplinaire HAL, est destinée au dépôt et à la diffusion de documents scientifiques de niveau recherche, publiés ou non, émanant des établissements d'enseignement et de recherche français ou étrangers, des laboratoires publics ou privés. 


\title{
LARGE AMPLITUDE VIBRATIONS OF THIN HYPERELASTIC PLATES: NEO-HOOKEAN MODEL
}

\author{
Ivan Breslavsky \\ Marco Amabili* \\ Mathias Legrand
Department of Mechanical Engineering Department of Mechanical Engineering Department of Mechanical Engineering McGill University
Montreal, Quebec, H3A 0C3
Canada
McGill University
Montreal, Quebec, H3A 0C3
Canada
McGill University
Montreal, Quebec, H3A 0C3
Canada \\ Email: ivan.breslavskyi@mcgill.ca \\ Email: marco.amabili@mcgill.ca \\ Email: mathias.legrand@mcgill.ca
}

\begin{abstract}
Static deflection and large amplitude vibrations of a rubber plate are analyzed. Both the geometrical and physical (material) nonlinearities are taken into account. The properties of the plate hyperelastic material are described by the Neo-Hookean law. A method for building a local model, which approximates the plate behavior around a deformed configuration, is proposed. This local model takes the form of a system of ordinary differential equations with quadratic and cubic nonlinearities. The results obtained with the help of this local model are compared to the solution of the exact model, and are found to be accurate. The difference between the model retaining both physical and geometrical non-inearities and a model with only geometrical nonlinearities is also analyzed. It is found that influence of physically induced nonlinearities at moderate strains is significant.
\end{abstract}

\section{INTRODUCTION}

Structural elements of materials with nonlinear stress-strain relationship are widely used in engineering. From a practical point of view the most important classes of such materials are rubbers and biomaterials. Such elements can be subject to high dynamic loads, which leads to their significant deformations. In such problems not only geometrical nonlinearity (the nonlinearity of strain-displacements relations), but also physical or mate-

*Address all correspondence to this author. rial nonlinearity (nonlinearity of stress-strain relations) should be taken into account.

Stress-strain relations for rubbers and biomaterials are described by hyperelastic models [1]. However, due to the complicated nature of the proposed models, very few studies on dynamics of shell and plate structures made of hyperelastic materials are reported. Reviews of static and dynamic problems can be found in $[2,3]$. A majority of research studies on the dynamics of hyperelastic thin-walled structures deals with simple geometries (spherical and circular cylindrical shells, circular membranes) and assume the same simple shape of the deformed structure [4-12].

Only a few works use more advanced approach. Goncalves et al. $[3,13]$ investigated linear and nonlinear free and forced vibrations of pre-stretched annular hyperelastic membranes. The membrane material is assumed to be incompressible, homogeneous, isotropic, and described by Neo-Hookean constitutive law. The in-plane displacements are neglected while the transverse displacement field is approximated by a series of natural modes. In both studies it is found that a single degree-of-freedom model correctly predicts large vibrations. It is shown that (i) a lightly stretched membrane displays a highly nonlinear hardening response, (ii) the nonlinearity decreases as the stretching ratio increases, and (iii) the response becomes essentially linear for a deformed radius of at least twice the initial value. In reference [3], a comparison is conducted for different hyperelastic 
models such as the Mooney-Rivlin, Yeoh, Ogden and ArrudaBoyce models. The results show that the membrane exhibits the same nonlinear frequency-amplitude behaviour for all tested models, with just a slight difference for the Ogden model.

In a majority of the existing works a incompressible NeoHookean law is employed to describe the nonlinear elastic behavior of rubber: accordinlgy, this model is considered in the present paper which also extends hyperelastic plate finite amplitude bending vibrations to commonly ignored in-plane displacements. Also, the majority of papers use pre-assumed shape of deformation for the structure, and this is a significant simplification. Due to the internal resonances, higher modes can be involved in vibrations, making deformed plate or shell configuration quite complicated [14]. The present study is an attempt to overcome the above-mentioned restriction. The deformation of a rubber rectangular plate under static pressure is investigated and subsequent free and forced large-amplitude vibrations around the corresponding static equilibrium are analyzed. The displacements are approximated by truncated series of linear eigenmodes and the convergence of these series is explored. The comparison with only geometrically nonlinear model is carried out. A method for building a local model, which approximates the plate behavior around a deformed configuration, is proposed. This local model takes the form of a system of ordinary differential equations with quadratic and cubic nonlinearities.

\section{LAGRANGE EQUATIONS}

The Lagrange equations are used in order to describe the dynamic behavior of plate:

$$
\frac{\partial}{\partial t}\left(\frac{\partial L}{\partial \dot{q}_{i}}\right)-\frac{\partial L}{\partial q_{i}}=Q_{i},
$$

where $L=T-\Pi$ is the Lagrange's functional; $\Pi=\iiint_{V} W \partial V$ is the potential energy of elastic deformation; $W$ is the strain energy density; $Q_{i}$ are the generalized forces; $V$ is the volume of the plate; $T$ is the kinetic energy of the plate, which is given by [14]:

$$
T=\frac{1}{2} \rho h \iint_{S}\left(\dot{u}^{2}+\dot{v}^{2}+\dot{w}^{2}\right) \partial S,
$$

where $S$ is the surface of the middle plane of the plate; $\rho$ is the density of the plate material, $h$ is the thickness of the plate and $u, v, w$ are the displacements along the axes of the rectangular coordinate system $x, y, z$, respectively.

\section{GEOMETRICALLY NONLINEAR DISPLACEMENT RELATIONSHIPS}

STRAIN-

The geometrical nonlinearity is described by using the von Kármán nonlinear plate theory [14]. Therefore, the strain- displacement relationships are given by:

$$
\begin{aligned}
& \varepsilon_{1}=\frac{\partial u}{\partial x}+\frac{1}{2}\left(\frac{\partial w}{\partial x}\right)^{2}-z \frac{\partial^{2} w}{\partial x^{2}} \\
& \varepsilon_{2}=\frac{\partial v}{\partial y}+\frac{1}{2}\left(\frac{\partial w}{\partial y}\right)^{2}-z \frac{\partial^{2} w}{\partial y^{2}} \\
& \varepsilon_{12}=\frac{\partial u}{\partial y}+\frac{\partial v}{\partial x}+\frac{\partial w}{\partial x} \frac{\partial w}{\partial y}-2 z \frac{\partial^{2} w}{\partial x \partial y}
\end{aligned}
$$

where $\varepsilon_{1}, \varepsilon_{2}$, and $\varepsilon_{12}$ are the components of the Lagrange strain tensor for thin plates. The displacements are expanded into truncated series:

$$
\begin{aligned}
& w(x, y, t)=\sum_{i=1}^{N_{W}} q_{i}(t) W_{i}(x, y) \\
& u(x, y, t)=\sum_{i=1}^{N_{U}} q_{i+N_{W}}(t) U_{i}(x, y) ; \\
& v(x, y, t)=\sum_{i=1}^{N_{V}} q_{i+N_{W}+N_{U}}(t) V_{i}(x, y),
\end{aligned}
$$

where $W_{i}, U_{i}, V_{i}$ are the admissible functions that satisfy the homogeneous boundary conditions (i.e. the geometric constraints) of the problem. A good choice of admissible functions are the eigenmodes of linear vibrations, which are a complete set. The total number of degrees of freedom is given by $N=N_{W}+N_{U}+N_{V}$.

\section{PHYSICAL RELATIONS Strain energy density}

Static and dynamic deflection of a thin rubber plate is considered. The nonlinear elasticity of natural rubber is described by Neo-Hookean constitutive law. The strain energy density for Neo-Hookean law has the following form [1]:

$$
W=\frac{E}{4(1+v)}\left(\bar{I}_{1}-3\right)+\frac{E}{6(1-2 v)}(J-1)^{2},
$$

where $\bar{I}_{1}=I_{1} J^{-\frac{2}{3}} ; I_{1}$ is the first invariant of the right CauchyGreen deformation tensor $\mathbf{C} ; J$ is the square root of the third invariant of the right Cauchy-Green deformation tensor; $E, v$ are the Young's modulus and Poisson's ratio of plate material, respectively.

The results obtained with Neo-Hookean strain energy density (9) are compared to those obtained by using the linear strain energy density (i.e. linear elasticity). For thin plates, the linear 
strain energy density has the following expression [14]:

$$
W=\frac{E}{4(1+v)}\left(\frac{2}{1-v}\left(\varepsilon_{1}^{2}+\varepsilon_{2}^{2}+2 v \varepsilon_{1} \varepsilon_{2}\right)+\varepsilon_{12}^{2}\right) .
$$

In this physically linear case, spatial and temporal components of the solution can be uncoupled and the Lagrange equations are simple ordinary differential equations with quadratic and cubic nonlinearities [14]

$$
\begin{gathered}
\ddot{q}_{n}+2 \zeta_{n} \Omega_{n} \dot{q}_{n}+\sum_{i=1}^{N} k_{n i} q_{i}+\sum_{i, j=1}^{N} k_{n i j} q_{i} q_{j}+ \\
\sum_{i, j, l=1}^{N} k_{n i j l} q_{i} q_{j} q_{l}=Q_{n}, \quad n=1, \ldots, N
\end{gathered}
$$

where $\Omega_{n}$ is the natural frequency of mode $n ; \zeta_{n}$ is the corresponding damping ratio; $k_{n i}, k_{n i j}, k_{n i j l}$ are known coefficients, that result from integration in space and $Q_{n}$ are the generalized forces, obtained by differentiation of the virtual work $R$ done by external forces

$$
Q_{n}=\frac{\partial R}{\partial q_{n}}
$$

Equations of type (11) are well known and there are several techniques for their numerical solution [14].

\section{Invariants of the Cauchy-Green deformation tensor}

In order to derive the expressions of the right Cauchy-Green deformation tensor $\mathbf{C}$ invariants in terms of displacements, the Lagrange strain tensor is introduced:

$$
\mathbf{E}=\frac{1}{2}\left(\begin{array}{ccc}
2 \varepsilon_{1} & \varepsilon_{12} & 0 \\
\varepsilon_{12} & 2 \varepsilon_{2} & 0 \\
0 & 0 & 2 \varepsilon_{3}
\end{array}\right)
$$

Expressions of $\varepsilon_{1}, \varepsilon_{2}$, and $\varepsilon_{12}$ (but not $\varepsilon_{3}$ ) are given by equation (3), (4), and (5), respectivly. The right Cauchy-Green deformation tensor $\mathbf{C}$ is then defined as follows [15]:

$$
\mathbf{C}=2 \mathbf{E}+\mathbf{I}=\left(\begin{array}{ccc}
2 \varepsilon_{1}+1 & \varepsilon_{12} & 0 \\
\varepsilon_{12} & 2 \varepsilon_{2}+1 & 0 \\
0 & 0 & 2 \varepsilon_{3}+1
\end{array}\right)
$$

and its invariants are easily expressed in terms of the Lagrange strain tensor components:

$$
\begin{aligned}
& I_{1}=\operatorname{Tr}(\mathbf{C})=2\left(\varepsilon_{1}+\varepsilon_{2}+\varepsilon_{3}\right)+3 \\
& J^{2}=|\mathbf{C}|=\left(2 \varepsilon_{3}+1\right)\left(\left(2 \varepsilon_{1}+1\right)\left(2 \varepsilon_{2}+1\right)-\varepsilon_{12}^{2}\right) \\
& \bar{I}_{1}=I_{1} J^{-\frac{2}{3}}=\frac{2\left(\varepsilon_{1}+\varepsilon_{2}+\varepsilon_{3}\right)+3}{\sqrt[3]{\left(2 \varepsilon_{3}+1\right)\left(\left(2 \varepsilon_{1}+1\right)\left(2 \varepsilon_{2}+1\right)-\varepsilon_{12}^{2}\right)}} .
\end{aligned}
$$

\section{Transverse normal strain}

The expression of the transverse normal strain $\varepsilon_{3}$ with respect to the plate displacements $u, v$, and $w$ has to be determined to later be inserted into equations (15)-(17). Here the approach based on the incompressibility condition of the plate material is employed.

The incompressibility condition gives $J=1$ [1]. Hence, from equation (16), we obtain

$$
\varepsilon_{3}=\frac{1}{2\left(\left(2 \varepsilon_{1}+1\right)\left(2 \varepsilon_{2}+1\right)-\varepsilon_{12}^{2}\right)}-\frac{1}{2} .
$$

Since the Poisson ratio is $v=0.5$ for an incompressible material, the corresponding Neo-Hookean strain energy density (9) for thin plates becomes:

$$
W=\frac{E}{3}\left(\varepsilon_{1}+\varepsilon_{2}+\frac{1}{2\left(\left(2 \varepsilon_{1}+1\right)\left(2 \varepsilon_{2}+1\right)-\varepsilon_{12}^{2}\right)}-\frac{1}{2}\right) .
$$

After series expansion of equation (19) in the strain components the terms of strain powers up to the second coincide with those of expression for physically linear strain energy density (10). That is, for small strains, both theories give the same results, which means that these theories are consistent.

\section{LOCAL EXPANSION OF THE NEO-HOOKEAN STRAIN ENERGY DENSITY}

Expression (19) is not a polynomial in strains, which essentially complicates the investigation of the plate behavior. Computers can obtain solutions of nonlinear systems, resulting from non-polynomial potential energy, but only for low-dimensional models.

In order to simplify the analysis, we introduce a transformation of expression (19). In particular, we want to obtain governing equations in the form of ordinary differential equations with nonlinearities of order not higher than three. Such model will be able to describe the behavior of the plate only locally, around a certain configuration.

We assume that we know a certain deformed configuration, identified by the set of values for generalized coordinates $\mathbf{q}^{(0)}=$ 
$\left\{q_{i}{ }^{(0)}\right\}_{i=1, \ldots, N}$ around which a new deformed configuration $\mathbf{q}=$ $\left\{q_{i}\right\}_{i=1, \ldots, N}$ is calculated:

$$
\mathbf{q}=\mathbf{q}^{(0)}+\alpha \mathbf{q}^{(1)},
$$

where $\alpha \ll 1$ is a small parameter. The strain components can be expressed as:

$$
\varepsilon_{1}=\varepsilon_{1}^{(0)}+\alpha \varepsilon_{1}^{(1)} ; \varepsilon_{2}=\varepsilon_{2}^{(0)}+\alpha \varepsilon_{2}^{(1)} ; \varepsilon_{12}=\varepsilon_{12}^{(0)}+\alpha \varepsilon_{12}^{(1)} .
$$

In the expressions (21) all $\alpha$-dependent terms are included in $\alpha \varepsilon_{i}^{(1)}$. Note that $\varepsilon_{1}^{(0)}, \varepsilon_{2}^{(0)}, \varepsilon_{12}^{(0)}$ do not depend on the unknown generalized coordinates $\mathbf{q}^{(1)}$.

We expand equation (19) into a series in the small parameter $\alpha$, keeping terms up to the second power:

$$
\begin{aligned}
W\left(q^{(0)}\right)= & \frac{E}{3}\left(\left[\varepsilon_{1}^{(0)}+\varepsilon_{2}^{(0)}+\frac{1}{2 \Xi}-\frac{1}{2}\right]+\alpha\left(\varepsilon_{1}^{(1)}+\varepsilon_{2}^{(1)}+\frac{\kappa}{\Xi^{2}}\right)+\right. \\
& \left.\alpha^{2}\left(\frac{4 \kappa^{2}-\left(4 \varepsilon_{1}^{(1)} \varepsilon_{2}^{(1)}-\left(\varepsilon_{12}^{(1)}\right)^{2}\right) \Xi}{2 \Xi^{3}}\right)\right),
\end{aligned}
$$

where

$$
\begin{aligned}
& \Xi=\left(2 \varepsilon_{1}^{(0)}+1\right)\left(2 \varepsilon_{2}^{(0)}+1\right)-\left(\varepsilon_{12}^{(0)}\right)^{2} ; \\
& \kappa=\varepsilon_{1}^{(1)}+\varepsilon_{2}^{(1)}+2 \varepsilon_{1}^{(0)} \varepsilon_{2}^{(1)}+2 \varepsilon_{2}^{(0)} \varepsilon_{1}^{(1)}-\varepsilon_{12}^{(0)} \varepsilon_{12}^{(1)} .
\end{aligned}
$$

Note that the small parameter $\alpha$ is only formal. In the sequel, the additional deflection $\mathbf{q}^{(1)}$ is assumed to be small with respect to $\mathbf{q}^{(0)}$.

The expression in square brackets in (22) does not depend on the generalized coordinates and vanish during differentiation. All denominators in (22) do not depend on the unknown generalized coordinates $\mathbf{q}^{(1)}$ and equation (22) is thus polynomial in $q_{i}^{(1)}$. Once the spatial and temporal parts are separated, the Lagrange equations (11) take the following form:

$$
\begin{aligned}
& \ddot{q}_{n}^{(1)}+2 \zeta_{n} \Omega_{n} \dot{q}_{n}^{(1)}+\sum_{i=1}^{N} k_{n i}\left(\mathbf{q}^{(0)}\right) q_{i}^{(1)}+ \\
& \sum_{i, j=1}^{N} k_{n i j}\left(\mathbf{q}^{(0)}\right) q_{i}^{(1)} q_{j}^{(1)}+\sum_{i, j, l=1}^{N} k_{n i j l}\left(\mathbf{q}^{(0)}\right) q_{i}^{(1)} q_{j}^{(1)} q_{l}^{(1)}=Q_{n}
\end{aligned}
$$

for $n=1, \ldots, N$. These equations represent the local model, which describes the behavior of the plate around the deformed configuration $\mathbf{q}^{(0)}$.
Now we describe the steps used to solve a static problem. We assume that a static load is indicated by the single-value parameter $P$. We start from a certain known deformed configuration $\mathbf{q}^{(0)}$.

Step 1. For a given static configuration $\mathbf{q}^{(0)}$, static counterpart of (24) where $\ddot{\mathbf{q}}^{(1)}=\dot{\mathbf{q}}^{(1)}=\mathbf{0}$ through the strain energy density (22) is built. At this stage, a total of $N+1$ unknowns ( $N$ generalized coordinates in $\mathbf{q}^{(1)}$ plus the applied load $P$ ) are identified.

Step 2. By choice the first generalized coordinate is enforced to be $q_{1}{ }^{(1)}=H$ with $H \ll q_{1}{ }^{(0)}$. The number of equations equals the number of unknowns.

Step 3. The system of equations is solved using the NewtonRaphson method and generalized coordinates $q_{i}^{(1)}, i=$ $2, \ldots, N$ as well as the applied force $P$ are determined.

Step 4. The static configuration is updated through $\mathbf{q}^{(0)} \leftarrow$ $\mathbf{q}^{(0)}+\mathbf{q}^{(1)}$ before going back to Step 1 of the next iteration.

The iterations continue until a desired deflection is reached.

\section{EXACT LOW-DIMENSIONAL MODELS WITH BOTH MA- TERIAL AND GEOMETRIC NONLINEARITIES}

Here we describe the approach that allows to check the accuracy of the approximate numerical solution obtained with the 4-step procedure previously described. This approach consists in the numerical solution of the Lagrange equations (1) in the static case with strain energy density (19). Since the latter is a smooth function in the generalized coordinates, integration and differentiation commute:

$$
\frac{\partial}{\partial \mathbf{q}}\left(\iiint_{V} W \partial V\right)=\iiint_{V} \frac{\partial W}{\partial \mathbf{q}} \partial V=\mathbf{Q} .
$$

For a prescribed pressure $P$, the current numerical solution $\mathbf{q}$ is substituted into (25). Accordingly, $\frac{\partial W}{\partial q_{i}}$ becomes a function of the spatial coordinates only and it is possible to perform numerical three-dimensional integration of equation (25). We apply the Newton-Raphson method to solve this system. The solution obtained with the present approach is named the exact solution hereinafter.

\section{NUMERICAL EXAMPLE Static analysis}

As an example, a simply supported rectangular rubber plate illustrated in Fig. 1 is considered. It is defined on the following domain:

$$
V=\{x \in[0 ; a], y \in[0 ; b], z \in[-h / 2 ; h / 2]\}
$$


with the following geometrical parameters $a=0.1 \mathrm{~m}, b=$ $0.12 \mathrm{~m}, h=0.0005 \mathrm{~m}$, and material characteristics $v=0.5$, $E=10^{7} \mathrm{~Pa}, \rho=1100 \mathrm{~kg} / \mathrm{m}^{3}$. The associated stress-strain di-

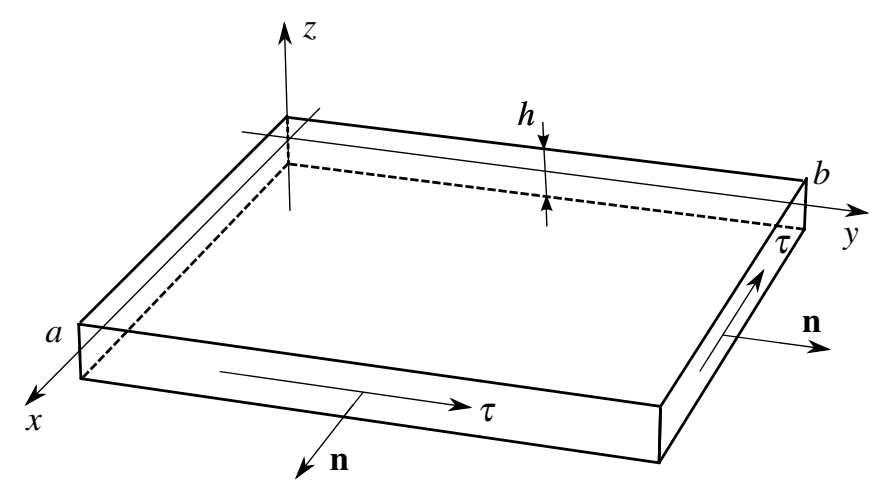

FIGURE 1: PLATE AND COORDINATE SYSTEM.

agram for uniaxial tension and compression is shown in Fig. 2.

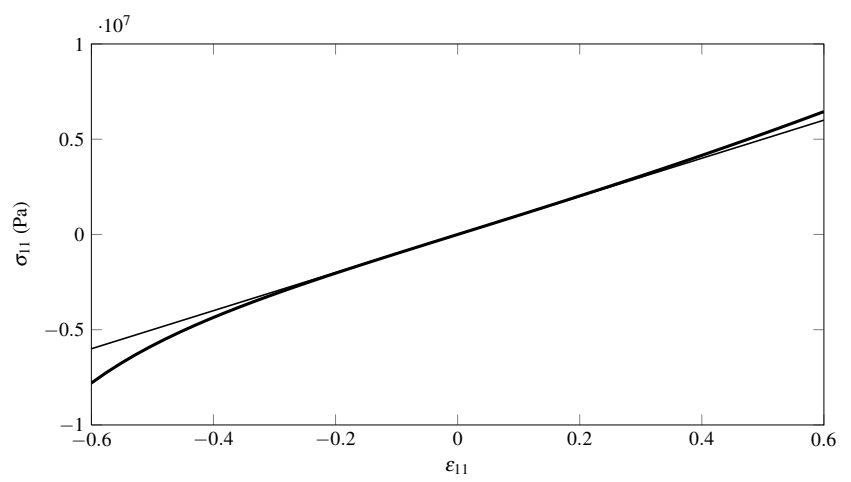

FIGURE 2: THE STRESS-STRAIN DIAGRAM FOR UNIAXIAL TENSION; CAUCHY STRESS: — NEO-HOOKEAN LAW; — HOOKES LAW.

The plate is simply supported with immovable edges, giving the following boundary conditions [14]:

$$
\left.w\right|_{\partial S}=\left.M\right|_{\partial S}=\left.u\right|_{\partial S}=\left.v\right|_{\partial S}=0
$$

where $\partial S$ denotes the boundary of the plate. The bending moment per unit length $M$ [14] reads:

$$
M=-D\left(\frac{\partial^{2} w}{\partial n^{2}}+v \frac{\partial^{2} w}{\partial \tau^{2}}\right)
$$

where $n$ and $\tau$ are the outer normal and tangent directions to $\partial S$, respectively. The corresponding linear eigenmodes are simple sine functions [14]:

$$
\begin{aligned}
& w(x, y, t)=\sum_{n, m \in \mathbb{N}} w_{n, m}(t) \sin \left(\frac{n \pi x}{a}\right) \sin \left(\frac{m \pi y}{b}\right) \\
& u(x, y, t)=\sum_{n, m \in \mathbb{N}} u_{n, m}(t) \sin \left(\frac{n \pi x}{a}\right) \sin \left(\frac{m \pi y}{b}\right) \\
& v(x, y, t)=\sum_{n, m \in \mathbb{N}} v_{n, m}(t) \sin \left(\frac{n \pi x}{a}\right) \sin \left(\frac{m \pi y}{b}\right) .
\end{aligned}
$$

Static and dynamic deflections under uniformly distributed constant pressure $P$ are now investigated. Due to symmetry considerations on the geometry of the plate and the distribution of the external load, only the odd bending modes are non-zero if no internal resonances are activated:

$$
\begin{aligned}
& w(x, y, t)= \\
& \sum_{n, m \in \mathbb{N}} w_{2 n+1,2 m+1}(t) \sin \left(\frac{(2 n+1) \pi x}{a}\right) \sin \left(\frac{(2 m+1) \pi y}{b}\right) .
\end{aligned}
$$

Respective in-plane modes participating in the solution have the form [14]:

$$
\begin{aligned}
& u(x, y, t)=\sum_{n, m \in \mathbb{N}} u_{2 n, 2 m+1}(t) \sin \left(\frac{2 n \pi x}{a}\right) \sin \left(\frac{(2 m+1) \pi y}{b}\right) \\
& v(x, y, t)=\sum_{n, m \in \mathbb{N}} v_{2 n+1,2 m}(t) \sin \left(\frac{(2 n+1) \pi x}{a}\right) \sin \left(\frac{2 m \pi y}{b}\right) .
\end{aligned}
$$

The problem is also appropriately scaled as detailed below:

$$
\begin{aligned}
\tau=\Omega_{1} t & \\
q_{i}=\frac{w_{2 n+1,2 m+1}}{h}, & i=1, \ldots, N_{W} ; \\
q_{i}=\frac{u_{2 n, 2 m+1}}{h}, & i=N_{W}+1, \ldots, N_{W}+N_{U} ; \\
q_{i}=\frac{v_{2 n+1,2 m}}{h}, & i=N_{W}+N_{U}+1, \ldots, N
\end{aligned}
$$

where $\Omega_{1}$ is the circular frequency of the first natural mode of the deformed plate. In the expresions (34) the two-indices generalized coordinates $w_{2 n+1,2 m+1}, u_{2 n, 2 m+1}, v_{2 n+1,2 m}$ are replaced by single-index coordinates $q_{i}$. For each particular problem, different terms have to be considered in the expansions (32) and (33) in order to have good accuracy.

Geometrically nonlinear bending. First, attention is paid to the strain energy density (10), where geometrical nonlinearities only are considered. The results are obtained for an 
increasing number of generalized coordinates in (32) and (33). In Fig. 3 the central deflection of the plate with respect to the

\begin{tabular}{ll}
\hline Size & Participating eigenmodes \\
\hline 3 DOFs & $w_{1,1}, u_{2,1}, v_{1,2}$ \\
$12 \mathrm{DOFs}$ & $w_{i, j}, i, j=1,3 ; u_{i, j}, v_{j, i}, i=2,4 ; j=1,3$ \\
$27 \mathrm{DOFs}$ & $w_{i, j}, i, j=1,3,5 ; u_{i, j}, v_{j, i}, i=2,4,6 ; j=1,3,5$ \\
$34 \mathrm{DOFs}$ & $w_{i, j}, i, j=1,3,5,7 ; u_{i, j}, v_{j, i}, i=2,4,6 ; j=1,3,5$ \\
\hline
\end{tabular}

TABLE 1: EIGENMODES USED FOR MODELS WITH DIFFERENT NUMBERS OF DOFS.

applied pressure is depicted. For each curve, participating eigenmodes are listed in Table 1.

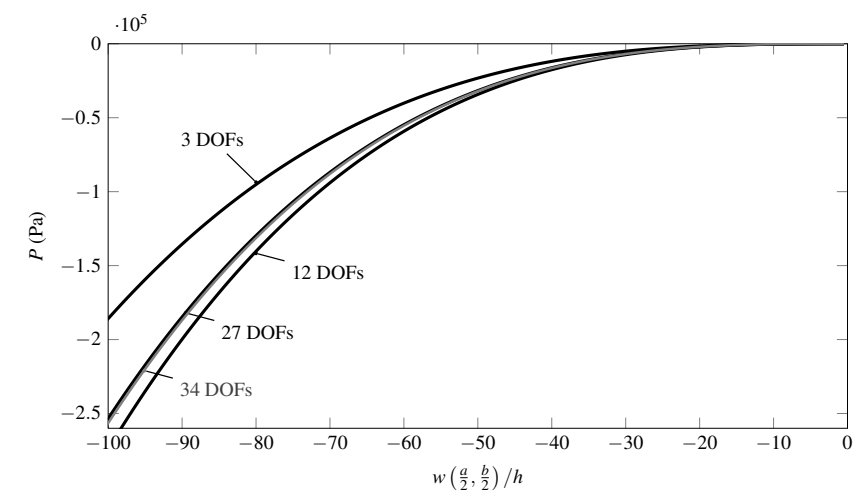

FIGURE 3: DEFLECTION-PRESSURE CURVES FOR MODELS WITH ONLY GEOMETRICAL NONLINEARITY.

The convergence is such that approximations approach the solution from alternating sides. The solution of the 27 DOFs system is between the solutions of the 3 DOFs and the 12 DOFs models, but closer to the 12 DOFs solution; the solution of the 34 DOFs system is between the solution of the 12 DOFs and the 27 DOFs systems, but closer to the 27 DOFs solution. The maximal difference between results of 12 and 34 DOFs models for a given pressure in the range of deflections $[0,100 h]$ is $2.2 \%$. So the 12 DOFs system gives results that are close enough to those of high-DOFs systems. In the following study we use the 12 DOFs system.
Physically and geometrically nonlinear bending. Exact model. The results for systems with 3, 12, 27 and 34 DOFs with both types of nonlinearities are obtained by the local model method and the strain energy density (9) is used. Also, the exact solutions are found for the 3 and 12 DOF systems. The participating modes in this subsection and in the remainder of the paper are the ones listed in Table 1.

The comparison of deflection-pressure curves for the model with only geometrical nonlinearity and exact solution for the 12 DOFs systems is shown in Fig. 4. We can see that the difference within a range of deflection up to $30 \mathrm{~h}$ is small. Only a $6 \%$ maximal difference for a given pressure is observed in this range.

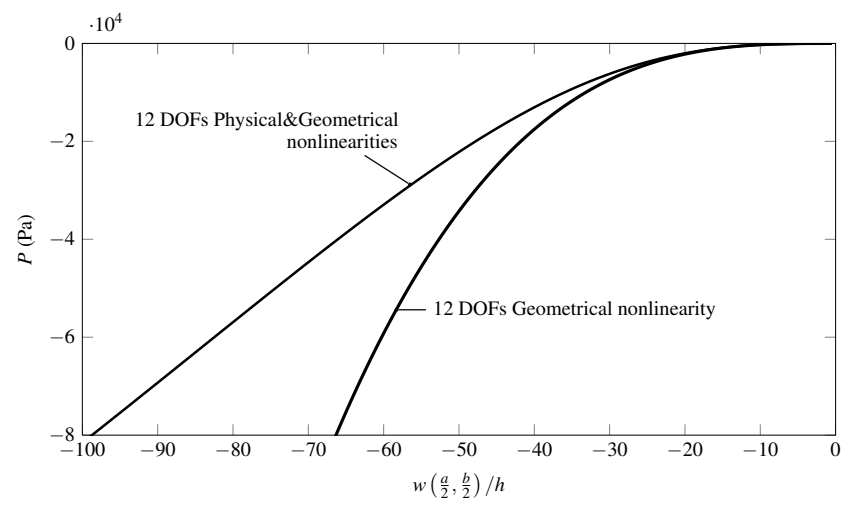

FIGURE 4: COMPARISON OF THE EXACT DEFLECTIONPRESSURE CURVES FOR THE MODEL WITH ONLY GEOMETRICAL AND BOTH GEOMETRICAL AND PHYSICAL NONLINEARITIES; 12 DOFS.

Local model. As displayed in Fig. 4, for deflections up to 30h, the model with only geometrical nonlinearity satisfactorily approximates the solution involving both nonlinearities. Therefore numerical iterations to obtain deflection curves are started at deformation of $30 \mathrm{~h}$ in Fig. 5 and only exact solutions are shown for smaller deflection.

Fig. 5 compares the corresponding results with both nonlinearities and an increasing number of DOFs to the available exact solutions. It is shown that the local model provides a sufficiently good approximation of the underlying plate behavior. Again, the 12 DOF model stands as a convincing compromise between prediction capabilities and computational cost. The maximal difference in deflection in the range of deflection $[0,100 h]$ is $2 \%$ with respect to the 34 DOF model. 


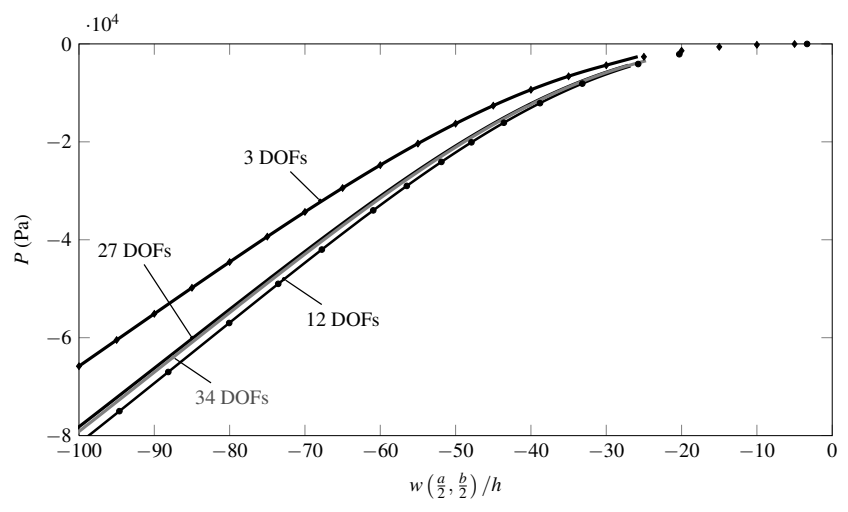

FIGURE 5: DEFLECTION-PRESSURE CURVES FOR MODELS WITH PHYSICAL AND GEOMETRICAL NONLINEARITIES:- , — OBTAINED WITH LOCAL MODELS; 3 DOFS EXACT SOLUTION; • 12 DOFS EXACT SOLUTION.

\section{Dynamic analysis}

Since at small strains the effect of physical nonlinearities can be neglected, we study the free and forced vibrations around a pre-loaded state. We chose as the initial deformed configuration the one with principal generalized coordinate $w_{1,1}=80 h$. The comparison with the exact static solution shows that local model around this deformed configuration is accurate for deflection up to $10 \mathrm{~h}$, so we limit our dynamical analysis to this amplitude.

For vibration analysis the 12 DOFs model is used.

Free and forced vibrations. The harmonic balance method [16] is applied to obtain periodic solutions of the system of type (11) through a Fourier expansion of the generalized coordinates:

$$
q_{i}(\tau)=A_{i, 0}+\sum_{j=1}^{N_{h}} A_{i, j} \cos (j \Omega \tau), \quad i=1, \ldots, 12
$$

where $\Omega$ is the non-dimensional frequency, normalized with respect to $\Omega_{1}$. Harmonics $A_{i, j}$ are determined from the system of nonlinear algebraic equations, that results from balancing of coefficients associated to the same harmonics in equations (11) after substituting equation (35). A convergence analysis shows that $N_{h}=8$ in (35) is the sufficient number of harmonics for a good approximation of the solution.

Fig. 6 displays the backbone curves for free vibrations with frequencies close to the first eigenfrequency of the pre-loaded plate. Non-dimesional frequencies, normalized with respect to the natural frequency $\Omega_{1}$ of the deflected plate are shown in abscissa.

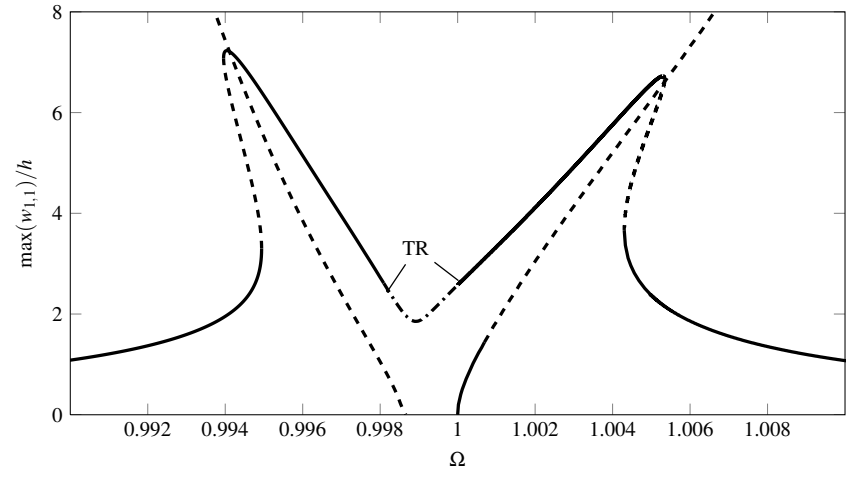

FIGURE 6: FREQUENCY RESPONSES AND BACKBONE CURVES FOR PRINCIPAL BENDING MODE $w_{1,1}:$ - STABLE MOTIONS; - - - UNSTABLE MOTIONS; - - - QUASIPERIODIC MOTIONS; TR, NEIMARK-SACKER BIFURCATION. $P_{d}=26.5 \mathrm{~Pa}, \zeta=0.001$.

The analysis of linearized system (11) shows that the first eigenfrequency of the deformed plate is equal to $2302.12 \mathrm{rad} / \mathrm{s}$ which is 86.626 times greater than first eigenfrequency of the initial flat plate.

Stable and unstable orbits are shown by solid and dashed lines, respectively. Stability analysis is based on calculation of frequency multipliers $[16,17]$.

It is well-known that the frequency-deflection relationship for simply supported flat plates is highly nonlinear [18-20]. However, the deformed plate under analysis exhibits very weak nonlinearity (the difference between frequency of vibrations with amplitude $10 \mathrm{~h}$ and linear eigenfrequency is only $0.8 \%$ ), but the behavior of plate is not that of a simple linear oscillations. There is an internal resonance 2:1 with in-plane mode $v_{1,2}$ (softening branch in Fig. 6), which can be observed from the linear modal responses analysis. Interaction with in-plane modes is observed in both backbone curves, shown in Fig. 6. This effect of weaken nonlinearity in stretched membranes is reported in $[3,13]$. For the study of forced vibrations the AUTO software [21] is employed. In Fig. 6 the forced vibration response to dynamic pressure excitation $P_{d}=26.5 \mathrm{~Pa}$, with damping ratio $\zeta_{n}=\zeta=0.001$, is shown. The response presents two peaks, the first one being associated to the 2:1 internal resonance, and two Neimark-Sacker bifurcations at $\Omega=0.998$ and $\Omega=1.000$. The forced vibration response in the frequency range between the two NeimarkSacker bifurcations is quasi-periodic, i.e. it presents amplitude modulations.

In order to verify the harmonic balance solution, the exact system of equations:

$$
\frac{\partial}{\partial t}\left(\frac{\partial T}{\partial \dot{\mathbf{q}}}\right)+\iiint_{V} \frac{\partial W}{\partial \mathbf{q}} \partial V=\mathbf{Q}
$$


is numerically integrated through the Runge-Kutta timemarching technique for free vibrations response. Prescribed initial displacements are obtained from the truncated Fourier series (35) at $\tau=0$. Initial velocities are zero.

\section{CONCLUSIONS}

A method for the analysis of large-amplitude vibrations of plates, taking into account physical and geometrical nonlinearities, is proposed. This method transforms the Lagrange equations in space and time into a system of ordinary differential equations in time only. This facilitates the investigation of the vibrations around a deformed configuration taking into account both physical and geometrical nonlinearities.

The method is tested on a thin rectangular rubber plate. The problems of static bending under pressure and free and forced vibrations near deformed configuration are studied. Both static and dynamic results, obtained by the present method, show good agreement with exact solutions.

While at small strains the influence of material nonlinearity is weak, at finite strains the effect of material nonlinearity is very significant. The deflection under given pressure is essentially underestimated by the model without material nonlinearity.

Differently from the case of oscillations near undeformed plate configuration, which shows strong amplitude-frequency dependence, the vibration frequency response around deformed plate configuration is close to linear. However, some nonlinear effects like bifurcations and internal resonances are present in the system.

\section{REFERENCES}

[1] Ogden, R., 1997. Non-Linear Elastic Deformations. Dover Publications, New York.

[2] Fu, Y. B., and Ogden, R. W., 2001. Nonlinear Elasticity: Theory and applications. Cambridge University Press, Cambridge.

[3] Goncalves, P. B., Soares, R. M., and Pamplona, D., 2009. "Nonlinear vibrations of a radially stretched circular hyperelastic membrane". Journal of Sound and Vibration, 327(12), pp. 231-248.

[4] Knowles, J. K., 1960. "Large amplitude oscillations of a tube of incompressible elastic material". Quarterly of Applied Mathematics, 18, pp. 71-77.

[5] Knowles, J. K., 1962. "On a class of oscillations in the finite-deformation theory of elasticity". Journal of Applied Mechanics, 29(2), pp. 283-286.

[6] Akkas, N., 1978. "On the dynamic snap-out instability of inflated non-linear spherical membranes". International Journal of Non-Linear Mechanics, 13(3), pp. 177-183.

[7] Verron, E., Khayat, R. E., Derdouri, A., and Peseux, B.,
1999. "Dynamic inflation of hyperelastic spherical membranes". Journal of Rheology, 43(5), pp. 1083-1097.

[8] Akyuz, U., and Ertepinar, A., 1998. "Stability and asymmetric vibrations of pressurized compressible hyperelastic cylindrical shells". International Journal of Non-Linear Mechanics, 34(3), pp. 391-404.

[9] Ju, Y., and Niu, D., 2012. "On a class of differential equations of motion of hyperelastic spherical membranes". Applied Mathematical Sciences, 6(81-84), pp. 4133-4136.

[10] Ren, J.-S., 2008. "Dynamical response of hyper-elastic cylindrical shells under periodic load". Applied Mathematics and Mechanics (English Edition), 29, pp. 1319-1327.

[11] Yuan, X., Zhang, R., and Zhang, H., 2008. "Controllability conditions of finite oscillations of hyper-elastic cylindrical tubes composed of a class of ogden material models". Computers, Materials and Continua, 7, pp. 155-166.

[12] Yuan, X.-G., Zhang, H.-W., Ren, J.-S., and Zhu, Z.-Y., 2010. "Some qualitative properties of incompressible hyperelastic spherical membranes under dynamic loads". Applied Mathematics and Mechanics (English Edition), 31, pp. 903-910.

[13] Soares, R. M., and Goncalves, P. B., 2012. "Nonlinear vibrations and instabilities of a stretched hyperelastic annular membrane". International Journal of Solids and Structures, 49(3-4), pp. 514-526.

[14] Amabili, M., 2008. Nonlinear vibrations and stability of shells and plates. Cambridge University Press, New York.

[15] Bower, A., 2010. Applied mechanics of solids. CRC Press. Taylor and Francis Group, Boca Raton.

[16] Parker, T. S., and Chua, L. O., 1989. Practical numerical algorithms for chaotic systems. Springer-Verlag, New York.

[17] Yakubovich, V., and Starzhinskii, V., 1975. Linear differential equations with periodic coefficients. Wiley, New York.

[18] Amabili, M., 2003. "Theory and experiments for largeamplitude vibrations of empty and fluid-filled circular cylindrical shells with imperfections". Journal of Sound and Vibration, 262(4), pp. 921 - 975.

[19] Amabili, M., 2004. "Nonlinear vibrations of rectangular plates with different boundary conditions: theory and experiments". Computers and Structures, 82(31-32), pp. $2587-2605$.

[20] Breslavsky, I. D., 2012. "Stress distribution over plates vibrating at large amplitudes". Journal of Sound and Vibration, 331(12), pp. 2901-2910.

[21] Doedel, E. J., Champneys, A. R., Fairgrieve, T. F., Kuznetsov, Y. A., Sandstede, B., and Wang, X., 1998. AUTO 97: Continuation and Bifurcation Software for Ordinary Differential Equations (with HomCont). Concordia University, Montreal. 